\title{
Neutron-proton pairing in Nuclear Matter
}

\author{
Xiao-Hua Fan, ${ }^{1}$ Xin-le Shang,,${ }^{1, *}$ Jian-Min Dong, ${ }^{1}$ and Wei Zuo ${ }^{1,2}$ \\ ${ }^{1}$ Institute of Modern Physics, Chinese Academy of Sciences, Lanzhou 730000, China \\ ${ }^{2}$ School of Nuclear Science and Technology, \\ University of Chinese Academy of Sciences, Beijing 100049, China
}

\begin{abstract}
The self-energy effect on the neutron-proton (np) pairing gap is investigated up to the third order within the framework of the extend Bruecker-Hartree-Fock (BHF) approach combined with the BCS theory. The self-energy up to the second-order contribution turns out to reduce strongly the effective energy gap, while the renormalization term enhances it significantly. In addition, the effect of the three-body force on the np pairing gap is shown to be negligible. To connect the present results with the np pairing in finite nuclei, an effective density-dependent zero-range pairing force is established with the parameters calibrated to the microscopically calculated energy gap.
\end{abstract}

PACS numbers: 21.65.Cd, 26.60.-c, 74.20.Fg, 74.25.-q

*shangxinle@impcas.ac.cn 


\section{INTRODUCTION}

About 60 years ago, the importance of pairing correlation in nuclear systems were realized 11. Since then, a large number of experimental data have been accumulated, supporting the isovector $(\mathrm{T}=1)$ pairing between like nucleons [2, 3]. However, no clear evidence supports the isoscalar $(\mathrm{T}=0)$ pairing [4], despite of the fact that the $\mathrm{T}=0$ interaction is much stronger than the $\mathrm{T}=1$ interaction [5]. The main suppression of the $\mathrm{T}=0$ pairing might result from the strong spin-orbit splitting [6, 7]. And the recent calculations on the Gamow-Teller transition also suggest that the $\mathrm{T}=0$ pairing interaction plays a decisive role for the concentration of Gamow-Teller strength when the spin-orbit splitting becomes small [8].

On the other hand, the microscopic calculation of the $\mathrm{T}=0$ neutron-proton (np) pairing with bare nucleon-nucleon interactions in nuclear matter predicts a sizable energy gap with the magnitude of $12 \mathrm{MeV}$ [9 12], which seems too large to reconcile with the empirical information available from finite nuclei [13]. However, the microscopical predictions can be significantly changed via a refinement of the theoretical framework, such as considering the energy dependence of the self-energy [14, 15], including the relativistic effect 16], embodying the polarization effect [17] and so on. In particular the polarization effect [18 22] in nuclear medium has been shown to enhance or quench the $\mathrm{T}=1$ neutron-neutron (nn) pairing gap depending on the nuclear environment. As for the $\mathrm{T}=0 \mathrm{np}$ pairing, the recent paper [23] indicates that the polarization effect exhibits much less significant effect for symmetric nuclear matter at densities above the half of the saturation density. At low densities, it remains difficult and an open problem. Another significant re-scaling of the pairing gap in symmetric nuclear matter may come from the dressing of nucleons in an interacting system, which modifies the density of state and the effective energy gap [24, 25]. These modifications result from the energy denpendence of the single-particle (s.p.) self-energy $\Sigma(k, \omega)$. The reduction

of the gap due to the appearance of a quasiparticle strength $Z$ factor is up to about fifty percent for $\mathrm{T}=1 \mathrm{nn}$ pairing in pure neutron matter [24, 25], while it may become as large as about seventy percent for $\mathrm{T}=0 \mathrm{np}$ pairing in symmetric nuclear matter [14, 15].

In Ref. [14], within the framework of the Brueckner theory, the effect of the energydependent self-energy $\Sigma(k, \omega)$ has been studied. However, the self-energy is calculated only to the lowest-order approximation $M_{1}(k, \omega)$. As is known that the imaginary part of $M_{1}(k, \omega)$ goes to zero below the Fermi energy and the imaginary part of the rearrangement term 
$M_{2}(k, \omega)$ presents the contrary behavior ( $\operatorname{Im} M_{2}$ goes to zero above the Fermi energy) [2628]. The imaginary part of the self-energy also plays an important role in predicting the energy gap [14]. Therefore a more complete investigation by including the effect of the rearrangement contribution $M_{2}(k, \omega)$ is necessary in the study of the $\mathrm{T}=0 \mathrm{np}$ pairing within the extended BHF approach. In addition, the three-body force (3BF) is expected to enhance the ${ }^{3} P F_{2}$ nn pairing at high densities [29], but its effect on the $\mathrm{T}=0 \mathrm{np}$ pairing has not been reported yet. A more accurate estimate of the np pairing gap should include the $3 \mathrm{BF}$ effect.

In this work, we shall concentrate on the modification of the gap equation including the energy dependence of the single-particle self-energy $\Sigma(k, \omega)$ up to the third-order approximation within the extended BHF approach. Moreover, the effect of $3 \mathrm{BF}$ on the ${ }^{3} S D_{1} \mathrm{np}$ pairing is also considered. The paper is organized as follows: In Sec. II, we briefly review the self-energy within the extended BHF approach and the formalism of the off-shell BCS gap equation. The numerical results and discussion are shown in Sec. III, where an effective density-dependent zero-range pairing force is provided with the parameters fitted to the calculated energy gap. And finally a summary is given in Sec. IV.

\section{SELF-ENERGY WITHIN THE EBHF APPROACH AND THE OFF-SHELL GAP EQUATION}

The present calculation of the self-energy $\Sigma(k, \omega)$ for symmetric nuclear matter is based on the extended BHF approach, for which one can refer to Ref. [27] for more details. The microscopic 3BF supplement to the extended BHF scheme can be found in Refs. [30, 31]. After several self-consistent iterations, the effective interaction matrix $G$ in the BrueckerBethe-Goldstone (BBG) theory is obtained. Using the G-matrix, the self-energy $\Sigma(k, \omega)$ can be calculated.

\section{A. Self-energy up to the third order}

Within the framework of the BBG theory, the self-energy $\Sigma(k, \omega)$ can be expanded in a perturbation series according to the number of hole lines [32]. The expansion up to the thirdorder contribution is shown diagrammatically in Fig.1. To the lowest-order approximation 


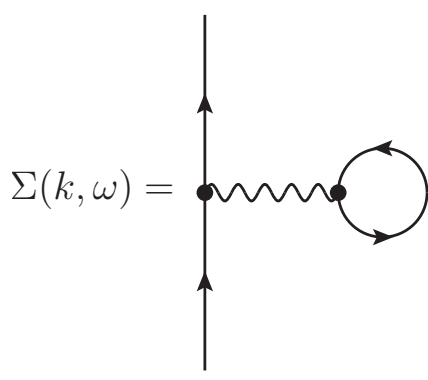

(a)

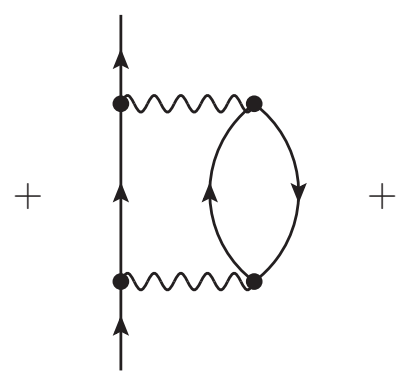

(b)

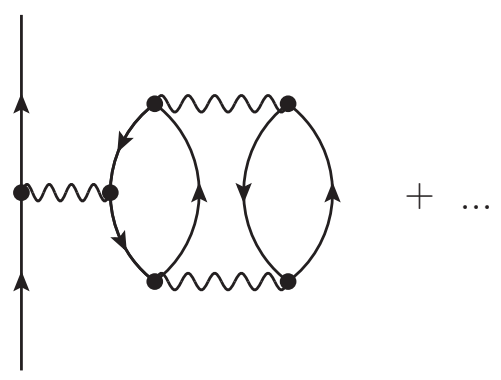

$(c)$

FIG. 1: Hole-line expansion of the self-energy.

in the hole-line expansion, i.e., the BHF approximation, the self-energy is written as

$$
M_{1}(k, \omega)=\sum_{k^{\prime}} n\left(k^{\prime}\right)\left\langle k k^{\prime}\left|G\left[\omega+\epsilon\left(k^{\prime}\right)\right]\right| k k^{\prime}\right\rangle_{A},
$$

where $\epsilon(k)$ is the s.p. energy spectrum in the BHF approximation and $\omega$ is the starting energy. $n(k)$ is the Fermi distribution function, which reduces to step function $\theta\left(k-k_{F}\right)$ at zero temperature. The subscript $A$ denotes antisymmetrization of the matrix elements.

The next order in the hole-line expansion of the self-energy, which is called rearrangement term, is given by [26]

$$
M_{2}(k, \omega)=\frac{1}{2} \sum_{k^{\prime} k_{1} k_{2}}\left[1-n\left(k^{\prime}\right)\right] n\left(k_{1}\right) n\left(k_{2}\right) \times \frac{\left|\left\langle k k^{\prime}\left|G\left[\epsilon\left(k_{1}\right)+\epsilon\left(k_{2}\right)\right]\right| k_{1} k_{2}\right\rangle_{A}\right|^{2}}{\omega+\epsilon\left(k^{\prime}\right)-\epsilon\left(k_{1}\right)-\epsilon\left(k_{2}\right)-i 0} .
$$

The corresponding diagrammatic sketch is shown in Fig.1 (b). It is related to the particlehole excitations in nuclear matter.

The third-order contribution in the expansion, as displayed in Fig.1 (c), accounts for the fact that hole state $h^{\prime}$ below the Fermi surface is are partially unoccupied due to nucleonnucleon short-range correlations. Therefore, this contribution to the s.p. spectrum is called the renormalization contribution given by [26, 33]

$$
M_{3}(k, \omega)=\sum_{h^{\prime}} \kappa_{2}\left(h^{\prime}\right)\left\langle k h^{\prime}\left|G\left[\omega+\epsilon\left(k^{\prime}\right)\right]\right| k h^{\prime}\right\rangle_{A},
$$

with the lowest order of the depletion of the Fermi sea

$$
\kappa_{2}\left(h^{\prime}\right)=-\left.\left[\frac{\partial}{\partial \omega} M_{1}\left(h^{\prime}, \omega\right)\right]\right|_{\omega=\epsilon\left(h^{\prime}\right)},
$$

where $h^{\prime}$ refers to the hole state satisfy the condition $\left|\overrightarrow{h^{\prime}}\right| \leq k_{F} . \kappa_{2}\left(h^{\prime}\right)$ is the probability that a hole state is empty. An estimate of $\kappa_{2}\left(h^{\prime}\right)$ consists in using the average value of the 
depletion, which is $\kappa=\kappa_{2}\left(h^{\prime}=0.75 k_{F}\right)$ [27, 33]. Then the renormalization contribution $M_{3}(k, \omega)$ can be estimated by $M_{3}(k, \omega) \approx \kappa M_{1}(k, \omega)$. Ref. [33] also shows that $\kappa \sim 0.25$ for symmetric nuclear matter in the density range $\rho \in\left(0.4 \rho_{0}, 2.3 \rho_{0}\right)$, where $\rho_{0}=0.17 \mathrm{fm}^{-3}$ is the empirical saturation density, indicating the non-negligible effect of $M_{3}$.

\section{B. The off-shell gap equation and approximation}

Generally, the four-dimensional gap equation including the self-energy $\Sigma(k, E)$ can be written as [34 34$]$

$$
\Delta(k, E)=\int \frac{d^{3} k^{\prime}}{(2 \pi)^{3}} \int \frac{d E^{\prime}}{2 \pi i} \mathcal{V}\left(k, E ; k^{\prime}, E^{\prime}\right) \Gamma\left(k^{\prime}, E^{\prime}\right) D\left(k^{\prime}, E^{\prime}\right),
$$

where the energy $E$ is defined as the energy relative to the chemical potential $\mu$, i.e., $E=$ $\omega-\mu$. And the kernel $\Gamma$ is defined as

$$
\begin{aligned}
\Gamma(k, E) & =\mathcal{G}(k,-E) \mathcal{G}^{s}(k, E) \\
& =\left[\mathcal{G}^{-1}(k, E) \mathcal{G}^{-1}(k,-E)+\Delta^{2}(k, E)\right]^{-1},
\end{aligned}
$$

with

$$
\begin{aligned}
\mathcal{G}(k, E) & =\left[E-\frac{k^{2}}{2 m}-\Sigma(k, \mu+E)+\mu+i 0 \text { sign } E\right]^{-1}, \\
\mathcal{G}^{s}(k, E) & =\frac{1}{G^{-1}(k, E)+\Delta^{2}(k, E) G(k,-E)} .
\end{aligned}
$$

The functions $\mathcal{G}(k, E)$ and $\mathcal{G}^{s}(k, E)$ are the nucleon propagators in the normal state and in the superfluid state for symmetric nuclear matter, respectively. We stress that the neutron propagator differs only slightly from the proton propagator due to the charge-dependent interaction and we ignore this difference in this paper. The symmetry of $E$ in the kernel $\Gamma$ is attributed to the time-reversal invariance of the Cooper pairs.

In principle, the pairing interaction $\mathcal{V}\left(k, E ; k^{\prime}, E^{\prime}\right)$ should include the polarization corrections. In this paper, the energy-independent interaction kernel is given merely by the bare two-body potential or by the bare two-body interaction plus a microscopic 3BF. Accordingly, the energy gap $\Delta$ is energy independent as well. To be more precise, the angle-averaged gap [10, 12, 38, 39] equation in the ${ }^{3} S D_{1}$ channel, which actually has a couple channel structure involving a two-component gap equation [40], can be expressed as,

$$
\left(\begin{array}{c}
\Delta_{0} \\
\Delta_{2}
\end{array}\right)(k)=\frac{1}{\pi} \int_{0}^{\infty} k^{\prime 2} d k^{\prime}\left(\frac{1}{\pi} \int_{0}^{\infty} d E \operatorname{Im} \Gamma\left(k^{\prime}, E\right)\right) \times\left(\begin{array}{cc}
V^{00} & V^{02} \\
V^{20} & V^{22}
\end{array}\right)\left(k, k^{\prime}\right)\left(\begin{array}{c}
\Delta_{0} \\
\Delta_{2}
\end{array}\right)\left(k^{\prime}\right),(9)
$$


where $V_{L L^{\prime}}\left(k, k^{\prime}\right)$ are the matrix elements of the bare interaction in the relevant coupled channels $\left(L, L^{\prime}=0,2\right)$ and the total gap corresponds to $\Delta^{2}(k)=\Delta_{0}^{2}(k)+\Delta_{0}^{2}(k)$.

Resolving this gap equation exactly requires the knowledge of the real and imaginary parts of the self-energy at arbitrary energy $E$ and momentum $k$. A strong simplification may be reached by assuming a small imaginary part of $\Sigma, \operatorname{Im} \Sigma \approx 0$, which leads to a quasiparticle approximation [28, 41, 42]. In this approximation the kernel $\Gamma(k, E)$ is an even function of energy: there exist two symmetric poles $\pm \Omega_{k}$, corresponding to the quasiparticle spectra in the superfluid state, on the real axis of the complex $E$ plane. The integral over the energy range in the Eq. (9) can be performed as follow:

$$
\frac{1}{\pi} \int_{0}^{\infty} d E \operatorname{Im} \Gamma(k, E)=-\frac{\mathcal{Z}_{k}^{2}}{2 \Omega_{k}}
$$

where the residue $\mathcal{Z}_{k}^{2}$ of the kernel at the pole $\Omega_{k}$ corresponds to the quasiparticle strength which is given by

$$
\begin{aligned}
\mathcal{Z}_{k}^{-2} & =\left.\left(\frac{\partial \mathcal{G}^{-1}(k, E)}{\partial E}\right)\right|_{E=E_{k}} \times\left.\left(\frac{\partial \mathcal{G}^{-1}(k,-E)}{\partial E}\right)\right|_{E=-E_{k}} \\
& =\left.\left[1-\frac{\partial \Sigma(k, \mu+E)}{\partial E}\right]^{2}\right|_{E=E_{k}} .
\end{aligned}
$$

The spectra of the single particle in normal state and the quasiparticle in the BCS state are expressed as

$$
\begin{aligned}
E_{k} & =\frac{k^{2}}{2 m}+\Sigma\left(k, \mu+E_{k}\right)-\mu \\
\Omega_{k} & =\sqrt{E_{k}^{2}+\mathcal{Z}_{k}^{2} \Delta^{2}(k)} .
\end{aligned}
$$

Then the gap equation can be approximated by

$$
\left(\begin{array}{c}
\Delta_{0} \\
\Delta_{2}
\end{array}\right)(k)=-\frac{1}{\pi} \int_{0}^{\infty} k^{\prime 2} d k^{\prime}\left(\begin{array}{cc}
V^{00} & V^{02} \\
V^{20} & V^{22}
\end{array}\right)\left(k, k^{\prime}\right) \frac{\mathcal{Z}_{k^{\prime}}^{2}}{2 \sqrt{E_{k^{\prime}}^{2}+\mathcal{Z}_{k^{\prime}}^{2} \Delta^{2}\left(k^{\prime}\right)}}\left(\begin{array}{c}
\Delta_{0} \\
\Delta_{2}
\end{array}\right)\left(k^{\prime}\right)
$$

Note that the effective energy gap is $\mathcal{Z}_{k} \Delta(k)$ instead of $\Delta(k)$ due to the dispersive self-energy [41] which is also true for the exact gap of equation (9). The presence of the quasiparticle strength, which is less than unitary in a small region around the Fermi surface where the Cooper pairs are mainly formed, reduces the pairing gap.

The gap equation should be solved self-consistently with the density constraint since the pairing could modify the chemical potential when the density is fixed in symmetric nuclear 
matter. In the superfluid state, the density can be expressed as

$$
\rho=4 \sum_{k} \int_{-\infty}^{0} \frac{d E}{\pi} \operatorname{Im} G^{s}(k, E),
$$

where a factor of four comes from the spin and isospin degrees of freedom. In this paper the numerical investigation is based on a self-consistently solution of the two coupled gap equations, Eqs.(9) and (14). The self-energy is considered up to the third order in the hole-line expansion.

\section{RESULTS AND DISCUSSIONS}

The numerical calculation here focuses on the ${ }^{3} S D_{1}$ np paring gap with inclusion of the self-energy effect and the 3BF effect. The realistic Argonne $V 18$ two-body interaction is adopted as the pairing interaction which is consistent with the self-energy calculated within the framework of the extended BHF approach using the same interaction. And the microscopic 3BF adopted here is constructed by using the meson-exchange current approach as in Refs. [30, 31].

As an illustrative example, the real and imaginary parts of the off-shell self-energy $\Sigma(k, \omega)$ at a density of $0.1 \mathrm{fm}^{-3}\left(k_{F}=1.14 \mathrm{fm}^{-1}\right)$ and a fixed momentum value of $k=0.85 \mathrm{fm}^{-1} \approx$ $0.75 k_{F}$ is exhibited in the upper panel of Fig.2. As mentioned in the introduction, the imaginary part of $\Sigma(k, \omega)$ goes to zero at the Fermi energy. This is true for the momentum $k=k_{F}$ as well, which implies the quasiparticle strength approximation is reliable near $k_{F}$. However, $\operatorname{Im} \Sigma$ becomes sizable compared to the real part of the self-energy at the s.p. energy when $k$ apart from $k_{F}$, and the imaginary part should be handled seriously. The lower panel of Fig.2 shows the real parts of $M_{2}$ and $M_{3}$. The magnitude of $\operatorname{Re} M_{3}$ is even larger than that of $\operatorname{Re} M_{2}$. Consequently, a reliable prediction of the self-energy effect requires to account for the third-order contribution $M_{3}$ and the second-order contribution $M_{2}$ at the same footing.

The knowledge of $\Sigma(k, \omega)$ allows us to solve the gap equations (9) and (14) exactly. Fig.3 displays the effective energy gaps $\mathcal{Z}_{F} \Delta_{F}\left(\Delta_{F}=\Delta\left(k_{F}\right)\right)$ at the Fermi momentum $k_{F}$. In the calculated result denoted by $M 1+M 2$ with $3 \mathrm{BF}$, the $3 \mathrm{BF}$ contributions are embodied in both the self-energy and the pairing interaction. It is shown that the 3BF effect on the ${ }^{3} S D_{1}$ np pairing is insignificant which is consistent with the weak effect of $3 \mathrm{BF}$ on the equation of state of nuclear matter at low density. The calculated results in the two cases 


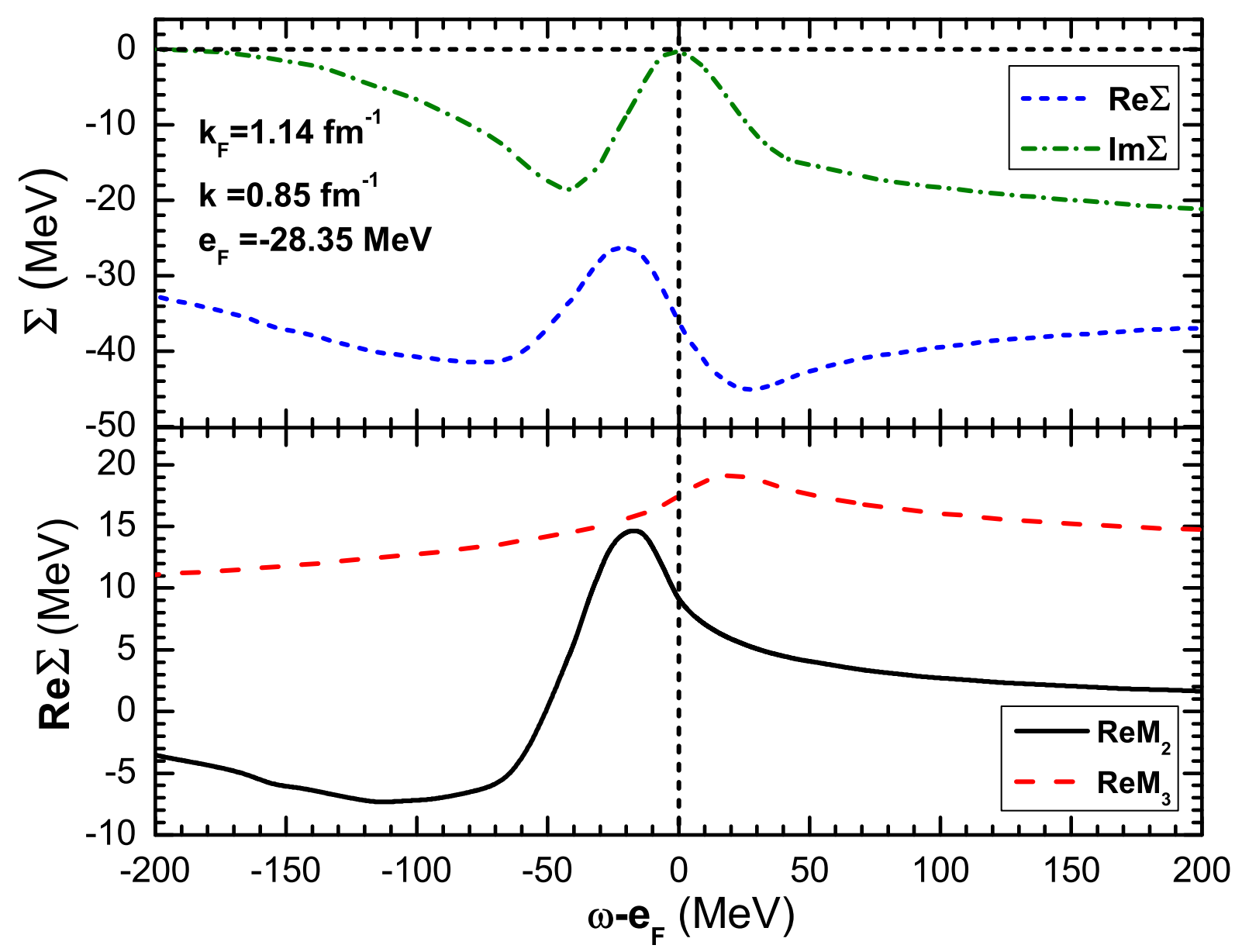

FIG. 2: (Color online). The real and imaginary parts (upper panel) of the self-energy $\Sigma$ and the the rearrangement and renormalization terms (lower panel) of the self-energy $\Sigma$ for $k=0.85 \mathrm{fm}^{-1}$ at $k_{F}=1.14 \mathrm{fm}^{-1}$. The short-dashed vertical line indicates the position of the Fermi energy.

with and without $M_{3}$ by adopting the two-body force only (the 3BF effect is negligible) are also compared in Fig.3. The locations of the maximum $\mathcal{Z}_{F} \Delta_{F}$ with and without $M_{3}$ are almost the same and around the density $0.055 \mathrm{fm}^{-3}\left(k_{F}=0.93 \mathrm{fm}^{-1}\right)$. The behaviors of $\mathcal{Z}_{F} \Delta_{F}$ with and without $M_{3}$ as functions of density are nearly-identical as well. However, the magnitude of the effective energy gap with $M_{3}$ is about two times of that without $M_{3}$. The self-energy up to the second-order approximation leads to a very strong reduction of 


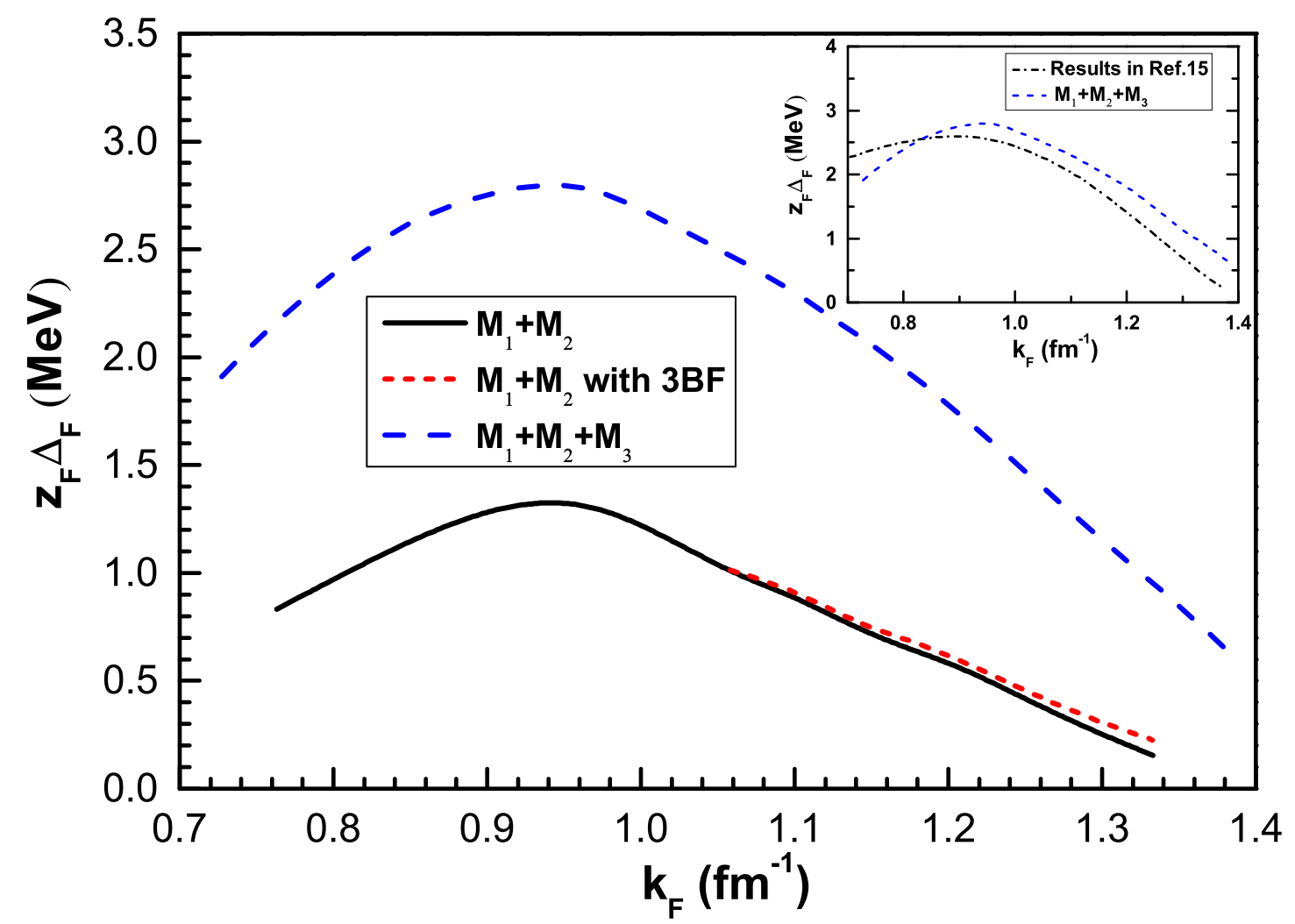

FIG. 3: (Color online). Neutron-proton effective energy gap in symmetric nuclear matter vs the Fermi momentum $k_{F}$. The black solid, red short dashed and blue dashed lines correspond to $M_{1}+M_{2}$ with $2 \mathrm{BF}, M_{1}+M_{2}$ with $3 \mathrm{BF}$ and $M_{1}+M_{2}+M_{3}$ with $2 \mathrm{BF}$, respectively. The inset compares the predictions in Ref. [15] with the calculated result denoted by $M_{1}+M_{2}+M_{3}$.

the ${ }^{3} S D_{1}$ np pairing gap, and makes the pairing gap even smaller than the nn pairing gap in pure neutron matter [42]. Nevertheless, the renormalization term of $\Sigma$ modifies both the quasiparticle strength and the density of state, which finally enhances the paring gap significantly.

In addition, a comparison between the present calculated results within the extended BHF approach and the predictions within the self-consistent in medium $\mathrm{T}$ matrix approximation [15] is shown in the inset. The present obtained effective gap in the extended BHF approach turns out to be slightly larger than that in the T matrix approximation in the density region of $\rho>0.045 \mathrm{fm}^{-3}$, while it becomes a bit smaller than that in the $\mathrm{T}$ matrix approximation 


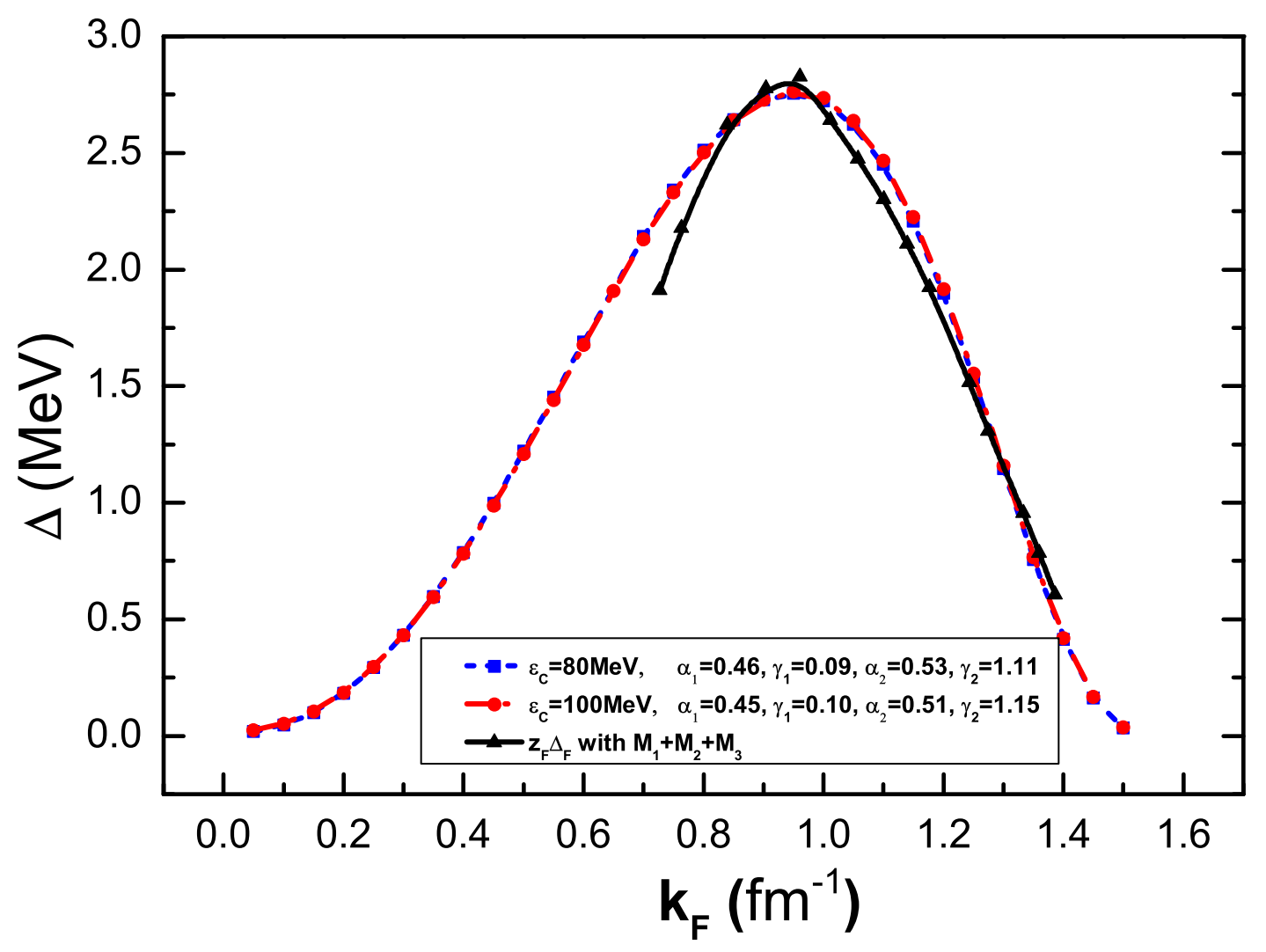

FIG. 4: (Color online). The np pairing gap using the effective density-dependent zero range pairing force and the calculated effective energy gap data within the EBHF approach.

when $\rho<0.045 \mathrm{fm}^{-3}$. In the extended BHF approach, the maximal effective gap is located at $0.055 \mathrm{fm}^{-3}$ with a value of $2.8 \mathrm{MeV}$, and the effective gap $\mathcal{Z}_{F} \Delta_{F} \approx 0.75 \mathrm{MeV}$ at the saturation density. However, in the T matrix approximation, the maximal effective gap is located at $0.05 \mathrm{fm}^{-3}$ with a value of $2.6 \mathrm{MeV}$, and the effective gap $\mathcal{Z}_{F} \Delta_{F} \approx 0.45 \mathrm{MeV}$ at the saturation density. Both calculations reveal a strong reduction of the effective pairing gap at saturation density due to the effect of the self-energy. The relativistic effect has been considered in Ref. [16] and it may also lead to a strong suppression of the pairing gap at saturation density.

To make contact with the pairing correlations in finite nuclei, we propose an effective density-dependent zero-range pairing force which include the reduction effect from the selfenergy. The parameters of the effective pairing force are determined to reproduce the cal- 
culated gap values. We propose an effective pairing force as follow:

$$
V_{\text {pairing }}\left(\mathbf{r}_{1}, \mathbf{r}_{2}\right)=v_{0}\left[1-\alpha_{1}\left(\frac{\rho\left(\frac{\mathbf{r}_{1}+\mathbf{r}_{2}}{2}\right)}{\rho_{0}}\right)^{\gamma_{1}}\right]\left[1-\alpha_{2}\left(\frac{\rho\left(\frac{\mathbf{r}_{1}+\mathbf{r}_{2}}{2}\right)}{\rho_{0}}\right)^{\gamma_{2}}\right] \delta\left(\mathbf{r}_{1}-\mathbf{r}_{2}\right)
$$

where $\rho_{0}=0.17 \mathrm{fm}^{-3}$ is the saturation density of symmetric nuclear matter and $v_{0}, \alpha_{1}, \gamma_{1}, \alpha_{2}, \gamma_{2}$ are parameters which is adjusted to reproduce the present predicted gap in symmetric nuclear matter. Being different from the proposed effective density-dependent zero-range pairing force in Ref. [43], we add an additional density-dependent factor $\left[1-\alpha_{1}\left(\frac{\rho\left(\frac{\mathbf{r}_{1}+\mathbf{r}_{2}}{2}\right)}{\rho_{0}}\right)^{\gamma_{1}}\right]$ which is expected to take into account the effect of $\Sigma$ on the pairing gap. The exact physical picture of this additional term is not clear, and it can improve the fitting which is exhibited in Fig.4 where the values of the obtained parameters are also given. As is well known, the zero-range pairing force must supplement a cutoff $\varepsilon_{\mathrm{c}}$. But in principle, the two parameters $v_{0}$ and $\varepsilon_{\mathrm{c}}$ are not independent and their values should be chosen in such a way that the deuteron binding energy is reproduced at zero density limit. Under this constraint, the two parameters are determined to ensure the gap equations with a solution of $\mu \rightarrow-1.12 \mathrm{MeV}$ and a finite gap value when $\rho \rightarrow 0$. With this constraint, the values of the parameter $v_{0}=-502.77 \mathrm{MeV}$ and $v_{0}=-440.73 \mathrm{MeV}$ correspond to $\varepsilon_{\mathrm{c}}=80 \mathrm{MeV}$ and $\varepsilon_{\mathrm{c}}=100 \mathrm{MeV}$, respectively. The two groups of parameters corresponding to the two different cutoffs produce nearly the same gap values. It is worth noticing the shape of the pairing gap as a function of $k_{F}$ (related to the density) in Fig.4 looks similar to the behavior of the pairing gaps for ${ }^{1} S_{0}$ channel. In fact, except for the different dominant density regions and magnitudes of the pairing gaps, the shapes of the pairing gaps in ${ }^{1} S_{0}$ and ${ }^{3} S D_{1}$ channels behave quite similar. As is known, the pairing correlation stems mainly from the attraction of nuclear interaction at Fermi momentum $k_{F}$. The attractions of both ${ }^{1} S_{0}$ and ${ }^{3} S D_{1}$ interactions at $k_{F}$ depends essential on density. And the density behavior of both attractions are quite similar, i.e., first increases up to a certain density and then decreases with the density.

\section{SUMMARY AND OUTLOOK}

In conclusion, we have investigated the self-energy effect on the ${ }^{3} S D_{1}$ np pairing gap within the extended BHF approach plus BCS theory. The rearrangement and renormalization terms of self-energy are considered. The self-energy up to the second-order approxima- 
tion presents a strong reduction of the effective energy gap, while the renormalization term enhances the pairing gap significantly. The maximum effective energy gap is located at the density $\rho=0.055 \mathrm{fm}^{-3}$ with a value of $2.8 \mathrm{MeV}$. The $3 \mathrm{BF}$ effect on the np pairing gap is studied as well, and it is found to be ignorable. Furthermore, an effective density-dependent zero-range pairing force is proposed with the parameters fitted to the calculated energy gap.

In this paper, we have concentrated on the self-energy effect, and the polarization corrections to the pairing interaction are not considered. Since an exact treatment of the polarization effect is notoriously difficult due to its complicated, different approximations are adopted to discuss the polarization effects [21, 44]. It has been shown that the polarization effect is negative to the pairing gap at low densities in the one-bubble approximation, whereas it is slightly positive in the full RPA limit. Moreover, as mentioned in the introduction, in Ref. 23] it is indicated that the polarization corrections appear to be negligible for moderate densities, yet it is still unclear and an open problem at low density. An improvement of the present calculation is to include the polarization effect in the future.

\section{Acknowledgments}

The work is supported by National Natural Science Foundation of China (No. 11435014, 11505241,11775276), the 973 Program of China under No. 2013CB834405, the Youth Innovation Promotion Association of Chinese Academy of Sciences.

[1] A. Bohr, B.R. Mottelson, D. Pines, Phys. Rev. 100936 (1958).

[2] R. A. Broglia and V. V. Zelevinsky, Fifty Years of Nuclear BCS: Pairing in Finite Systems (World Scientific, 2013).

[3] D. J. Dean and M. Hjorth-Jensen, Rev. Mod. Phys. 75607 (2003).

[4] S. Frauendorf and A. O. Macchiavelli, Prog. Part. Nucl. Phys. 78 24-90 (2014).

[5] A. M. Lane, Nuclear Theory, Benjamin (1964).

[6] G. F. Bertsch and Y. Luo, Phys. Rev. C 81064320 (2010).

[7] H. Sagawa, C. L. Bai, and G. Colo, Phys. Scr. 91083011 (2016).

[8] K. Kaneko, Y. Sun, T. Mizusaki, Phys. Rev. C 97, 054326 (2018). 
[9] U.Lombardo, H.-J.Schulze, and W. Zuo, Phys. Rev. C. 59, 2927 (1999).

[10] Xinle Shang, and Wei Zuo, Phys. Rev. C. 88, 025806 (2013).

[11] T. Alm, G. Röpke, and M. Schmidt, Z. Phys. A 337355 (1990).

[12] Xin-le Shang, Pei Wang, Peng Yin and Wei Zuo, J. Phys. G: Nucl. Part. Phys. 42055105 (2015).

[13] G.Röpke, A.Schnell, P.Schuck, and U.Lombardo, Phys. Rev. C 61, 024306 (2000).

[14] M. Baldo, U. Lombardo, H. -J. Schulze, and Zuo Wei, Phys. Rev. C. 66, 054304 (2002).

[15] P. Bożek, Phys. Lett. B 55193 (2003).

[16] Ø.Elgaroy, L.Engvik, M.Hjorth-Jensen, and E.Osnes, Phys. Rev. C. 57, R1069 (1998).

[17] J. Clark, C.-G. Källman, C.-H. Yang, D. Chakkalakal, Phys. Lett. B 61331 (1976).

[18] J. Wambach, T.L. Ainsworth, D. Pines, Nucl. Phys. A 555128 (1993).

[19] H.-J. Schulze, J. Cugnon, A. Lejeune, M. Baldo, and U. Lombardo, Phys. Lett. B 3751 (1996).

[20] H.-J. Schulze, A. Polls, and A. Ramos, Phys. Rev. C 63044310 (2001).

[21] L. G. Cao, U.Lombardo, P.Schuck, Phys. Rev. C. 74, 064301 (2006).

[22] S. S. Zhang, L. G. Cao, U. Lombardo and P. Schuck, Phys. Rev. C 93044329 (2016).

[23] Wenmei Guo, U. lombardo and P. Schuck, Phys. Rev. C 99014310 (2019)

[24] M. Baldo, A. Grasso, Phys. Lett. B 485115 (2000).

[25] U. Lombardo, P. Schuck, W. Zuo, Phys. Rev. C 64021301 (2001).

[26] J. P. Jeukenne, A. Lejeune, and C. Mahaux, Phys. Rep., Phys. Lett. bf 25C 83 (1976).

[27] W. Zuo, I. Bombaci, and U. Lombardo, Phys. Rev. C 60024605 (1999).

[28] U. Lombardo, P. Schuck, and W. Zuo, Phys. Rev. C 64021301 (R) (2001).

[29] J. M. Dong, U. Lombardo and W. Zuo, Phys. Rev. C 87062801 (R) (2013).

[30] P. Grangé, A. Lejeune, M. Martzolff, and J. -F. Mathiot, Phys. Rev. C 401040 (1989).

[31] W. Zuo, A. Lejeune, U. Lombardo, and J.-F. Mathiot, Nucl. Phys. A 706418 (2002); Eur. Phys. J. A 14469 (2002).

[32] J. Hüfner and C. Mahaux, Ann. Phys. (N.Y.) 73525 (1972).

[33] M. Baldo, I. Bombaci, G. Giansiracusa, U. Lombardo, C. Mahaux and R. Sartor, Phys. Rev. C 411748 (1990).

[34] A. A. Abrikosov, L. P. Gorkov, and I. E. Dzyaloshinskii, Methods of Quantum Field Theory in Statistical Physics (Prentice- Hall, Englewood Cliffs, NJ, 1963). 
[35] P. Nozières, Theory of Interacting Fermi Systems (Benjamin, New York, 1966).

[36] A. B. Migdal, Theory of Finite Systems and Applications to Atomic Nuclei (Benjamin, New York, 1964).

[37] J. R. Schrieffer, Theory of Superconductivity (Addison-Wesley, New York, 1964).

[38] A. Sedrakian and U. Lombardo, Phys. Rev. Lett. 84, 602 (2000).

[39] A. Sedrakian, Phys. Rev. C. 63, 025801 (2001).

[40] M. Baldo, U. Lombardo, and P. Schuck, Phys. Rev. C 52975 (1995).

[41] P. Bożek, Phys. Rev. C 62054316 (2000).

[42] Caiwan Shen, U. Lombardo, P. Schuck, W. Zuo, and N. Sandulescu, Phys. Rev. C 67061302 (R) (2003).

[43] G. F. Bertsch and H. Esbensen, Ann. Phys. (N.Y.) 209, 327 (1991); H. Esbensen, G. F. Bertsch, and K. Hencken, Phys. Rev. C 56, 3054 (1997).

[44] Caiwan Shen, U. Lombardo, P. Schuck, Phys. Rev. C 71054301 (R) (2005). 